\title{
Resource Allocation For Reconfigurable Intelligent Surface Assisted Dual Connectivity
}

This paper was downloaded from TechRxiv (https://www.techrxiv.org).

\section{LICENSE}

CC BY 4.0

SUBMISSION DATE / POSTED DATE

$06-12-2021 / 10-12-2021$

\section{CITATION}

Ramamoorthi, Yoghitha; Iwabuchi, Masashi; Murakami, Tomoki; Ogawa, Tomoaki; Takatori, Yasushi (2021): Resource Allocation For Reconfigurable Intelligent Surface Assisted Dual Connectivity. TechRxiv. Preprint. https://doi.org/10.36227/techrxiv.17127038.v1

$\mathrm{DOI}$ 


\title{
Resource Allocation For Reconfigurable Intelligent Surface Assisted Dual Connectivity
}

\author{
Yoghitha Ramamoorthi, Masashi Iwabuchi, Tomoki Murakami, Tomoaki Ogawa, Yasushi Takatori
}

\begin{abstract}
The next generation 6G wireless systems are envisioned to have higher reliability and capacity than the existing cellular systems. The reconfigurable intelligent surfaces (RISs) assisted wireless networks are one of the promising solution to control the wireless channel by altering the electromagnetic properties of the signal. The dual connectivity (DC) increases the per-user throughput by utilizing radio resources from two different base stations. In this work, we propose the RIS assisted DC system to improve the per-user throughput of the users by utilizing resources from two base stations (BSs) in proximity via different RISs. Given an $\alpha-$ fair utility function, the joint resource allocation and the user scheduling of RIS assisted DC system is formulated as an optimization problem and the optimal user scheduling time fraction is derived. A heuristic is proposed to solve the formulated optimization problem with the derived optimal user scheduling time fractions. The exhaustive simulation results for coverage and throughput of the RIS assisted DC system are presented with varying user, BS, blockage, and RIS densities for different fairness values. Further, we show that the proposed RIS assisted DC system provides significant throughput gain of $52 \%$ and $48 \%$ in certain scenarios when compared to the existing benchmark and DC systems.
\end{abstract}

Index Terms-Base station, coverage, dual connectivity (DC), fairness, reconfigurable intelligent surface (RIS), resource allocation, RIS assisted DC, throughput, user scheduling.

\section{INTRODUCTION}

$\mathbf{T}$ HE increased requirements of network capacity and user data rate have been driving the $5 \mathrm{G}$ networks. The evolution of beyond $5 \mathrm{G}$ and the next generation $6 \mathrm{G}$ networks includes smart radio environment that can be achieved by controlling the wireless channel in a specified manner. The reconfigurable intelligent surface (RIS) is a promising solution to control the wireless channel by altering electromagnetic properties of the signal [1]. The RIS is also widely known as intelligent reflecting surfaces as it consists of the smaller antenna elements capable of reflecting the information signal with the particular phase. The phase and the reflection of the signal are actively controlled by the RIS. This capability makes the wireless channel controllable. Further, the RIS assisted wireless communication aids in improving the coverage area. The millimeter wave (mmWave) based cellular networks with high bandwidth are prone to high attenuation and pathloss. This property of mmWave networks makes it impossible to reach for long distance particularly if the user is in non line of sight (NLOS). Leveraging the advantage of RIS to control the channel in mmWave based cellular network is expected to improve the coverage and throughput of the users in high attenuation regions. Although, the RIS assisted wireless communication is in evolving stages, the appropriate resource allocation and scheduling schemes are still in the basic stages and needs thorough investigation.

The different analytical free-space path loss models based on the electromagnetic properties of RISs have been characterized in [2] for RIS assisted wireless communication systems. The comprehensive analysis of main operating principles of RISs and the current research challenges of these RISs have been discussed in detail in [3]. The far field path loss model using physics optics technique has been derived by authors in [4]. Further, they also explained how the small elements of the surface can be advantageous when jointly beamform the signal in the desired direction. Two types of implementation of
RIS based on reflect arrays or metasurfaces and its prospective channel modelling and path loss models have been discussed on [5]. The joint optimization of coordinated transmit beamforming at BS and the reflective beamforming vector at the RIS by maximizing signal-to-interference- plus-noise ratio (SINR) at the users subjecting to transmit power constraints have been considered in [6].

The RIS with multicell communication in order to avoid inter cell interference of the cell-edge users has been proposed in [7]. This inter cell interference has been eliminating by destructively combining the reflected signal from RIS. The multiple RISs aided wireless communication system where multiple RISs jointly assist the link between BS and cell edge users has been investigated in [8]. The joint RIS deployment and the design of reflection coefficients have been jointly considered in [9]. Some of the multiple access schemes like non orthogonal multiple access (NOMA), frequency division multiple access, and time division multiple access (TDMA) have also been considered during joint optimization in [9]. The joint optimization problem of channel, resource allocation, power allocation, and the reflection coefficients for a downlink (DL) RIS based NOMA has been investigated by authors in [10].

The RIS aided coordinated multipoint (CoMP) with joint processing has been proposed and discussed in [11] where the deployed RIS serves the cell-edge users by utilizing the signal from multiple BSs in proximity. The resource allocation for RIS aided vehicular communications based on slowly varying large-scale fading channel has been studied in [12]. The optimization of rate, energy efficiency, and their tradeoff in a RIS based system along with overhead estimation has been presented in [13]. The optimization of RIS passive beamforming and orthogonal frequency division multiple access (OFDMA) in a RIS based system has been proposed in [14]. A novel approach to enhance the achievable rate of a ODFM system using RIS has been presented in [15]. The detailed investigation of RIS assosited NOMA and orthogonal 
multiple access (OMA) schemes have been studied in [16]. A comprehensive comparision between RIS and relay based system over realistic mmWave channels has been presented in [17].

The Dual connectivity (DC) has been introduced in [18] for cellular networks to improve per-user rate. The user in DC utilizes radio resources from two BSs and serve the users. The are two BSs involved in DC. On is primary BS (PBS) where the user associated based on its maximum received power. The other one is secondary BS (SBS) where the user receives its second maximum SINR from. The eligible DC user is able to receive different traffic from these two BSs. The first step of this to ensure the amount of traffic routed from OBS to the user via SBS. The downlink traffic scheduling and the SBS traffic routing to SBS in DC has been derived and presented in [19]. The performance comparison between CoMP and DC based system for difference users and BS density scenarios have been presented in [20].

The RIS assisted wireless communication requires significant attention when it comes to the multi cell connectivity. The multi connectivity with multiple RIS assisted CoMP has been evaluated in literature [11]. The different scheduling and traffic routing schemes of DC also have been analyzed in [19] and [20]. The RIS assisted CoMP architectures are expected to improve coverage if the two RIS or BSs are in proximity. The CoMP in general requires the resources to be reserved for the CoMP users that gives rise to coverage throughput trade-off [21]. Most of the literature consider either RIS aided wireless system or DC system separately. To the best of our knowledge, this is the first work that proposes RIS assisted DC where the eligible user is expected to utilize radio resources from two BSs via two RIS deployed in proximity. Based on the above motivation, the following contribution are made

- The RIS assisted DC architecture is proposed which utilizes radio resources from two different BSs via two different RISs.

- The joint resource allocation of RIS assisted DC is formulated as an optimization problem and the optimal user scheduling time fraction for RIS assisted DC systems for an $\alpha$-fair scheduler is derived.

- The formulated joint problem is decomposed and the optimal user scheduling time fraction is derived using Karush-Kuhn-Tucker (KKT) conditions. A heuristic for solving overall optimization problem with the derived scheduling time fraction is also presented.

- The exhaustive simulation results are presented for distributed RIS architecture with varying densities of user, BSs, and blockage densities are presented.

The paper is organized as follows. The system model with various parameters is presented in Section. II. The joint problem formulation of RIS assisted DC is presented in Section. III. The heuristic for solving RIS assisted DC problem is presented in Section. IV. The evaluated numerical results are presented in Section. V. The conclusion and future work is given in Section. VI. The system model is presented next.

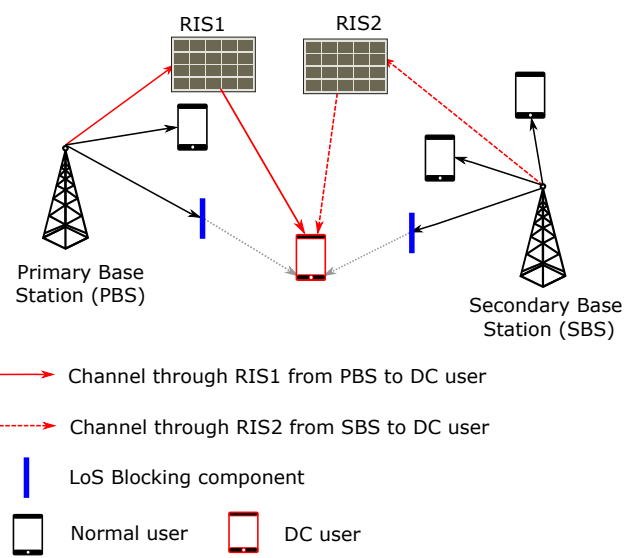

Fig. 1. System Model of RIS assisted DC.

TABLE I

MATHEMATICAL NOTATIONS

\begin{tabular}{|c|c|}
\hline$h_{u, j}^{0}$ & Direct channel gain at user $u$ from BS $j$ \\
\hline$\hat{h}_{u, j}^{r}$ & Channel gain at user $u$ from BS $j$ via RIS $r$ \\
\hline$l_{u, j}^{0}$ & Direct link rate of user $u$ from BS $j$ \\
\hline$l_{u, j}^{r}$ & Link rate of user $u$ from BS $j$ via RIS $r$ \\
\hline$x_{u, j}^{0}$ & $\begin{array}{c}\text { Binary user association variable of user } \\
u \text { with BS } j \text { withour RIS }\end{array}$ \\
\hline$x_{u, j}^{r}$ & \begin{tabular}{c}
$u$ with BS $j$ via RIS \\
\hline$\alpha$
\end{tabular} \\
\hline$\delta_{u, j}^{0}$ & Eser scheduling time fraction for user $u$ by BS $j$ without RIS \\
\hline$\delta_{u, j}^{r}$ & User scheduling time fraction for user $u$ by BS $j$ via RIS $r$ \\
\hline$\Gamma()$. & Spectral efficiency in bits $/$ symbol \\
\hline$\mu_{b}$ & Blockage density \\
\hline$\omega_{u, j}^{0}$ & DL received SINR of user $u$ from a BS $j$ \\
\hline$\omega_{u, j}^{r}$ & DL received SINR of user $u$ from a BS $j$ via RIS $r$ \\
\hline$\rho_{b s}$ & Number of BSs \\
\hline$\rho_{u s e r}$ & Number of users \\
\hline$\Lambda$ & Utility function \\
\hline$d_{u, j}^{r}$ & Binary association variable for DC user \\
\hline$\lambda_{u}$ & Data rate of user $u$ \\
\hline$\beta_{r, n}$ & Reflection amplitude of $n^{t h}$ antenna element of $r^{t h}$ RIS \\
\hline$\theta_{r, n}$ & Phase shift of of $n^{t h}$ antenna element of $r^{t h}$ RIS \\
\hline$T_{\alpha}$ & Throughput \\
\hline
\end{tabular}

\section{SYSTEM MODEL}

We consider OFDMA based cellular system. The overall system model of RIS assisted DC is shown in the Fig. 1 Let us denote the set of base stations (BSs) as $\boldsymbol{J}=\{1,2,3, \ldots, \mathrm{J}\}$ and the set of users as $\boldsymbol{U}=\{1,2,3, \ldots, \mathrm{U}\}$. The set of subchannels in the system is denoted by $\boldsymbol{C}=\{1,2,3, \ldots, \mathrm{C}\}$. The set of RIS in the system is denoted as $\boldsymbol{R}=\{0,1,2,3, \ldots, \mathrm{R}\}$. Whenever RIS is denoted as 0 , it is considered as line of sight (LOS) direct link between BS and the user, i.e., there is no RIS between BS $j$ and user $u$. We assume that the RIS has $N$ elements in the panel to reflect and coherently combines and beamforms the signal. The set of antenna elements in the RIS is denoted by $\boldsymbol{N}=\{1,2,3, \ldots, \mathbf{N}\}$. Every antenna element has its own reflection coefficient and phase shift. The mathematical notations used in this paper are tabulated in Table. I. The physical channel modelling is presented in next subsection. 


\section{A. Physical Channel Model}

We consider the time division duplexing (TDD) based system and we focus on downlink (DL) in this work. The direct channel gain $h_{u, j}^{0}$ between the BS $j$ and the user $u$ is given as follows. We denote $x_{u, j}^{0}$ as the binary association variable that is 1 , if the direct link exists between BS $j$ and the user $u$ and 0 , if it is not. The association $x_{u, j}^{0}$ is based on the maximum received power and it is given as

$$
x_{u, j}^{0}=\left\{\begin{array}{l}
1, \text { if } j=\arg \max _{j}\left\{P_{j} h_{u, j}^{0}\right\}, \\
0, \text { otherwise, } \forall u \in \boldsymbol{U}, \forall j \in \boldsymbol{J},
\end{array}\right.
$$

where, $P_{j}$ is the transmit power of BS $j$ and $h_{u, j}^{0}$ is the direct LOS channel gain between BS $j$ and the user $u$ as expressed below.

$$
h_{u, j}^{0}=10^{-\left(\frac{P L(u, j)+v+\xi-G_{j}-G_{u}}{10}\right)}
$$

where $P L(u, j)$ is the pathloss between user $u$ and $\mathrm{BS} j, v$ is the small scale fading loss, $\xi$ is the loss due to log-normal shadowing, $G_{j}$ is the transmit antenna gain, and $G_{u}$ is the gain of user antenna. The pathloss between $u$ and $j$ is determined by the appropriate radio access terminal. The direct link SINR of user $u$ from the BS $j$ is given as follows.

$$
\omega_{u, j}^{0}=\frac{P_{j}^{c} h_{u, j}^{0}}{\sum_{j^{\prime} \in \boldsymbol{J} \backslash j} P_{j^{\prime}}^{c} h_{u, j^{\prime}}^{0}+\sigma^{2}}, \forall u \in \boldsymbol{U},
$$

where, $\sum_{j^{\prime} \in \boldsymbol{B} \backslash b} P_{j^{\prime}}^{0} h_{u, j^{\prime}}^{0}$ is the interference from the other BSs, $P_{j}$ is the transmit power of BS $j$, and $\sigma^{2}$ is the noise power. The link rate and the scheduling of the system is presented next.

\section{B. Link Rate and Scheduling}

We consider flat fading channels where all subchannels and channels over elements have similar channel gains. We also consider adaptive modulation and coding scheme (MCS) as in Table II with the SINR as in (3). Let, $\Gamma($.$) denotes the$ spectral efficiency obtained from MCS. Given SINR as in (3) and spectral efficiency $\Gamma($.$) (bits/symbol), the direct link rate$ of user $u$ from BS $j$ is

$$
l_{u, j}^{0}=\frac{\Gamma\left(\omega_{u, j}^{0}\right) S C_{O F D M} S Y_{O F D M} \mathrm{C}}{T_{s c}}, j \in \boldsymbol{j}, c \in \boldsymbol{C},
$$

where, $S C_{O F D M}, S Y_{O F D M}$, and $T_{s c}$ represent the number of subcarriers per subchannel, number of symbol per subcarrier, and time duration of a subframe, respectively. Given the direct link rate $l_{u, r}^{0}$ as in (4), the net data rate of user $u$ from BS $j$ for a conventional direct link is given as follows.

$$
\lambda_{u}=\sum_{j \in \boldsymbol{J}} x_{u, j}^{0} \delta_{u, j}^{0} l_{u, j}^{0}, \forall u \in \boldsymbol{U},
$$

where, the user association $x_{u, j}^{0}$ as in (1), link rate $l_{u, j}^{0}$ as in (4), $\delta_{u, j}^{0}$ is the user scheduling time fraction for user $u$ by BS $j$. The $\delta_{u, j}^{0}$ for the for the direct channel between BS $j$ and user $u$ is given as

$$
\delta_{u, j}^{0}=\frac{\left(l_{u, j}^{0}\right)^{\frac{1-\alpha}{\alpha}}}{\sum_{u \in \boldsymbol{U}} x_{u, j}^{0}\left(l_{u, j}^{0}\right)^{\frac{1-\alpha}{\alpha}}}, \forall j \in \boldsymbol{J},
$$

where, $\alpha$ is the fairness parameter. The channel modelling parameters explained in (1)-(6) are related to direct channel parameters between BS $j$ and the user $u$. These does not involve the RIS as a intermediate node. The channel modelling and user scheduling parameters of RIS based links are explained in the next subsection

\section{RIS Channel Model and Scheduling}

The physical channel model of RIS based system consists of two channel links. One is the channel between BS $j$ and RIS $r$ denoted as $f_{r, j}^{n}$ and the other one is between RIS $r$ and user $u$ denoted as $g_{u}^{n, r}$. The super script $n$ implies the $n^{t h}$ reflective element. The overall channel between user $u$ and BS $b$ via RIS $r$ is the cascading of the above two channels along with the reflective parameter matrix of of RIS $(\Theta)$, i.e., $f_{r, j}^{n} \Theta g_{u}^{n, r}$. The SINR of user $u$ from BS $j$ via RIS $r$ is denoted by $\hat{\omega}_{u, j}^{r}$ as given below

$$
\omega_{u, j}^{r}=\frac{P_{j}\left(h_{u, j}^{0}+\boldsymbol{f}_{r, j}^{n} \Theta \boldsymbol{g}_{u}^{n, r}\right)}{\sum_{j^{\prime} \in \boldsymbol{J} \backslash j} P_{j^{\prime}} h_{u, j^{\prime}}^{0}+\sigma^{2}}, \forall u \in \boldsymbol{U}, \forall r \geq 1
$$

where, $h_{u, j}^{0}$ is the direct channel gain between BS $j$ and user $u, \Theta$ is the diagonal matrix containing phase shifts $\left(\theta_{r, n}\right)$ and reflection coefficient values $\left(\beta_{r, n}\right)$ of each element $n$ of RIS $r$. The $\sum_{j^{\prime} \in \boldsymbol{B} \backslash b} P_{j^{\prime}}^{0} h_{u, j^{\prime}}^{0}$ is the interference from the other BSs, $P_{j}$ is the transmit power of $\mathrm{BS} j$, and $\sigma^{2}$ is the noise power. The interference from the other RIS is negligible because the received signal is assumed to be coherently combined and beamformed towards the intended receiver $u$. When the diagonal matrix is expanded in terms of $\left(\theta_{r, n}\right)$ and $\left(\beta_{r, n}\right)$, the SINR equation is expressed as

$\omega_{u, j}^{r}=\frac{P_{j}\left(h_{u, j}^{0}+\sum_{n=1}^{N} f_{j}^{r, n} \beta_{r, n} e^{j \theta_{r, n}} g_{u}^{r, n}\right)}{\sum_{j^{\prime} \in \boldsymbol{J} \backslash j} P_{j^{\prime}}^{c} h_{u, j^{\prime}}^{0}+\sigma^{2}}, \forall u \in \boldsymbol{U}, \forall r \geq 1$

where, $N$ is the total number of antenna elements in RIS, $\beta_{r, n}$, and $\theta_{r, n}$ is the reflection coefficient and phase shift of $n^{t h}$ antenna element of $r^{t h}$ RIS, respectively. Similar to (4), if the user $u$ is associated with BS $j$ via RIS $r$, then the link rate would be a function of $\omega_{u, j}^{r}$ and is given as follows.

$$
l_{u, j}^{r}=\frac{\Gamma\left(\omega_{u, j}^{r}\right) S C_{O F D M} S Y_{O F D M} \boldsymbol{C}}{T_{s c}}, j \in \boldsymbol{j}, c \in \boldsymbol{C} .
$$

Given the link rate $r$ as in the above equation, the actual data rate of the user $u$ in the RIS system is expressed as

$$
\lambda_{u}=\sum_{j \in \boldsymbol{J}} \sum_{r \in \boldsymbol{R}} x_{u, j}^{r} \delta_{u, j}^{r} l_{u, j}^{r}, \forall u \in \boldsymbol{U},
$$

where, the user association $x_{u, j}^{0}$ as in (12), link rate $l_{u, j}^{r}$ as in (9), $\delta_{u, j}^{r}$ is the user scheduling time fraction for user $u$ by BS 
TABLE II

MODULATION AND CODING SCHEME [21].

\begin{tabular}{|c|c|c|c|c|c|c|c|c|c|c|c|c|c|c|c|}
\hline SINR Threshold (dB) & -6.5 & -4 & -2.6 & -1 & 1 & 3 & 6.6 & 10 & 11.4 & 11.8 & 13 & 13.8 & 15.6 & 16.8 & 17.6 \\
\hline Spectral efficiency (bits/symbol) & 0.15 & 0.23 & 0.38 & 0.60 & 0.88 & 1.18 & 1.48 & 1.91 & 2.41 & 2.73 & 3.32 & 3.9 & 4.52 & 5.12 & 5.55 \\
\hline
\end{tabular}

$j$ via RIS $r$. The $\delta_{u, j}^{r}$ from BS $j$ to user $u$ via RIS $r$ is given as

$$
\delta_{u, j}^{r}=\frac{\left(l_{u, j}^{r}\right)^{\frac{1-\alpha}{\alpha}}}{\sum_{u \in \boldsymbol{U}} \sum_{r \in \boldsymbol{R}} x_{u, j}^{r}\left(l_{u, j}^{r}\right)^{\frac{1-\alpha}{\alpha}}}, \forall j \in \boldsymbol{J} .
$$

where $\alpha$ is the fairness parameter, and $x_{u, j}^{r}$ is the user association between the BS $j$ and user $u$ via RIS $r$. This is expressed as

$$
x_{u, j}^{r}=\left\{\begin{array}{l}
1, \text { if } j=\arg \max _{j, r}\left\{\omega_{u, j}^{r}\right\}, \\
0, \text { otherwise, } \forall u \in \boldsymbol{U}, \forall j \in \boldsymbol{J}, \forall r \in \boldsymbol{R},
\end{array}\right.
$$

The performance metrics considered in RIS assisted DC system is considered next.

\section{Performance Metrics}

We consider coverage and $\alpha$-fair throughput as our performance metric in this work. The coverage is defined as the probability of user receiving SINR greater than the minimum threshold as in Table. III We consider $\alpha$-fairness in this work. The utility function of $\Lambda_{\alpha}(x)$ with respect to general variable $x$ is written as follows.

$$
\Lambda_{\alpha}(x)= \begin{cases}\frac{x^{1-\alpha}}{1-\alpha}, & \alpha>0, \alpha \neq 1, \\ \log (x) & \alpha=1 .\end{cases}
$$

where $\alpha$ is the fairness parameter. When $\alpha=1$, (13) leads to proportional fairness. As $\alpha$ increases, the worst case user's rate of the system improves. $\alpha \rightarrow \infty$ corresponds to max-min fairness where then minimum rate of the user in the system is maximized. The optimization problem formulated for RIS assisted DC system is presented in the next section.

\section{RIS AsSisted DuAl ConNectivity}

We consider $x_{u, j}^{r}$ as binary association variable that indicates whether user $u$ is associated with $j$ via RIS $r$ or not. The tuple $(u, r, j)$ in all the variables indicates the identification values of user, RIS, and BSs. When the value of $r$ is 0 , then there is no RIS participating in the link between the user $u$ and BS $j$. If $r$ is not equal to 0 , then the the user $u$ is served by BS $j$ via RIS $r$. We denote $d_{u, j}^{r}$ is the association variable between BS $j$ and user $u$ via RIS $r$. When $d_{u, j}^{r}=1$, then the user $u$ receives SINR greater than the threshold $\tau$ from two different BS via two different BSs. The DC association variable of user $u$ with BS $k$ via RIS $q$ denoted as $d_{u, k}^{q}$ is 1 only when its primary association $x_{u, k}^{r}$ is 1 with some other BS $j$ via RIS $r$. Given the channel $\left(h_{u, j}^{0}, f_{j}^{r, n}, g_{u, j}^{r, n}\right)$ and the SINR $\left(\omega_{u, j}^{r}\right)$, the overall optimization problem for an $\alpha$-fair utility function is presented as follows.

$$
\mathbf{P 1}: \max _{\substack{\beta_{r, n}, \theta_{r, n}, \delta_{u, j}^{r}, \tau, x_{u, j}^{r}, d_{u, j}^{r}}} \sum_{u \in \boldsymbol{U}} \Lambda_{\alpha}\left(\lambda_{u}\right),
$$

$$
\begin{aligned}
& \text { s.t } \lambda_{u}=\sum_{r \in \boldsymbol{R}} \sum_{j \in \boldsymbol{J}} x_{u, j}^{r}\left(l_{u, j}^{r} \delta_{u, j}^{r}\right. \\
& \left.+\sum_{r \in \boldsymbol{R}} \sum_{k \in \boldsymbol{J}} d_{u, k}^{r} l_{u, k}^{r} \delta_{u, k}^{r}\right), \forall u \in \boldsymbol{U}, \\
& \sum_{u \in \boldsymbol{U}} \sum_{r \in \boldsymbol{R}}\left(x_{u, j}^{r}+d_{u, j}^{r}\right) \delta_{u, j}^{r} \leq 1, \forall j \in \boldsymbol{J}, \\
& \sum_{r \in \boldsymbol{R}} \sum_{j \in \boldsymbol{J}}\left(x_{u, j}^{r}+d_{u, j}^{r}\right) \leq 1, \forall u \in \boldsymbol{U}, \\
& x_{u, j}^{r} \text { as in (12), } \\
& d_{u, k}^{q}=\left\{\begin{array}{c}
1, \text { if } k, q=\arg \max _{k \in \boldsymbol{J} \backslash\{j\}}\left\{\omega_{u, k}^{q}\right\}, \\
\omega_{u, j}^{r} \geq \tau, \omega_{u, k}^{q} \geq \tau, \\
\text { 0, otherwise, } \forall u \in \mathcal{U},
\end{array}\right. \\
& x_{u, j}^{r} \in\{0,1\}, \forall u \in \boldsymbol{U}, \forall j \in \boldsymbol{J}, \forall r \in \boldsymbol{R}, \\
& d_{u, j}^{r} \in\{0,1\}, \forall u \in \boldsymbol{U}, \forall j \in \boldsymbol{J}, \forall r \in \boldsymbol{R}, \\
& \delta_{u, j}^{r} \geq 0, \forall u \in \boldsymbol{U}, \forall j \in \boldsymbol{J}, \forall r \in \boldsymbol{R}, \\
& \omega_{u, j}^{r} \text { as in (8) }, l_{u, j}^{r} \text { as in (9), } \\
& \beta_{r, n} \in[0,1] \text {, } \\
& \theta_{r, n} \in[0, \pi] \text {, }
\end{aligned}
$$

where (14) is the maximization of $\alpha$-fair utility function defined in (13) with respect to RIS parameters amplitude reflection coefficient $\beta_{r, n}$, phase shift coefficient $\theta_{r, n}$, the user scheduling time fraction $\delta_{u, j}^{r}$, and the binary variables $x_{u, j}^{r}$, and $d_{u, j}^{r}$. The constraint in (15) specifies the actual data rate of the user $u$. If $u$ has the association only with its primary BS (PBS) via the RIS $r$, then only $x_{u, j}^{r}$ is 1 . However, if $u$ is a DC user via RIS $r$, then $d_{u, j}^{r}$ is also equal to 1 . Thus DC user's data rate is computed from both the terms as in (15). However, if association is between the BS $j$ and user $u$ without RIS, then only $x_{u, j}^{0}$ is 1 . This is because the user $u$ already has the strong direct LOS channel from BS $j$. The constraint in (16) specifies that the sum of time fraction allocated to all the users by any BS $j$ directly or via any RIS $r$ can not exceed 1. Further, the constraint in (17) shows that the user $u$ can be associated to one $j$, i.e., if it associated to primary BS (PBS) $j$, then $x_{u, j}^{r}=1$ or if it associates to the secondary BS (SBS) $j$, then $d_{u, j}^{r}=1$. Both $x_{u, j}^{r}$ and $d_{u, j}^{r}$ will not be 1 at the same time for the same BS $j$ and RIS $r$. The condition for user association with PBS and its associated RIS is given in the constraint (12). The user $u$ is associated to the PBS $j$ via the RIS $r$ (if $r=0$, without RIS) that has maximum SINR. Similarly, the condition for DC user association with its SBS $k$ based on the constraint (18). The user is qualified to be a DC user if its SINR from both PBS $\left(\omega_{u, j}^{r}\right)$ and SBS $\left(\omega_{u, k}^{q}\right)$ via different RIS should greater than the threshold $\tau$. The binary constrains of association variable are specified in (19) and (20), respectively. The positivity constraint on user 
scheduling time fraction through RIS $r$ is given in (21). Since the set of RIS $\boldsymbol{R}$ also contains the value 0 , this positivity constraint is ensured for the scheduling time fraction for the direct LOS link between BS $j$ and user $u$ also. The constraints on amplitude reflection coefficient $\beta_{r, n}$ and the phase shift $\theta_{r, n}$ of the $n^{t h}$ antenna element of $r^{t h}$ RIS are given in (22) and (23), respectively.

The optimization problem presented in (14) is mixed integer non linear programming (MINLP) and is difficult to solve for all variables at once. Therefore, (14) is decomposed into subproblems that can be solved independently. Given the channel, $f_{j}^{r, n}$ and $g_{u, j}^{r, n}$, the maximum rate is achieved by selecting $\theta_{r, n}=\angle h_{u, j}^{0}-\angle f_{j}^{r, n} g_{u, j}^{n}$ for each element $n$ [22]. We assume flat fading channel, i.e., the channel between BS $j$ and every element $n$ of the RIS $r$ is same. Therefore, the channel between BS $j$ and RIS $r$ is now denoted as $f_{j}^{r}$. Similarly, the channel between RIS $r$ and user $u$ as $g_{u, j}^{r}$ and the amplitude of the reflection coefficient as 1 . The entire RIS is now focusing towards a single user $u$. The modified SINR with the channel and the optimal phase shifts can be conclusively written as in [22] as

$$
\omega_{u, j}^{r}=\frac{P_{j}\left(\sqrt{h_{u, j}^{0}}+N \sqrt{f_{j}^{r} g_{u}^{r}}\right)^{2}}{\sum_{j^{\prime} \in \boldsymbol{J} \backslash j} P_{j^{\prime}} h_{u, j^{\prime}}^{0}+\sigma^{2}}, \forall u \in \boldsymbol{U}, \forall r \in \boldsymbol{R}, r \geq 1
$$

where $\sum_{j^{\prime} \in \boldsymbol{J} \backslash j} P_{j^{\prime}} h_{u, j^{\prime}}^{0}$ is the interference received by the user $u$ from other BSs, $P_{j}$ is the transmit power. Since the RIS parameters are optimized and the SINR is computed as in (24), the goal is now to determine the normal, RIS, and RIS assisted DC users based on the computed SINR values. Given the SINR of $u$ from BS $j$ via RIS $r$, the link rate of the given link $l_{u, j}^{r}$ is computed as in (9), the global optimization problem is decomposed and formulated as user scheduling problem as below.

$$
\begin{array}{r}
\text { P2 : } \max _{\delta_{u, j}^{r}} \sum_{u \in \boldsymbol{U}} \Lambda_{\alpha}\left(\lambda_{u}\right) \\
\text { s.t 15 }-21
\end{array}
$$

The problem in $\mathbf{P 2}$ is the maximization of $\alpha$-fair utility function given the association, SINR and the link rates. 25 is now a convex problem with respect to the user scheduling time fraction $\delta_{u, j}^{r}$ and it is solved using KKT conditions. The proposition for deriving optimal user schedulinng time fraction $\delta_{u, j}^{j}$ is presented next.

Proposition 1: Given the binary association of user $u$ with BS $j$ with RIS $(r=0)$ or without RIS $(r>1)$, the secondary association $d_{u, j}^{r}$ based on the next maximum received SINR, the link rate of user $u$ with $\mathrm{BS} j$ as a normal or DC user, the user scheduling time fraction allocated to the user $u$ by $\mathrm{BS} j$ with or without RIS is given as follows.

$$
\delta_{u, j}^{r}=\frac{\left(x_{u, j}^{r}+d_{u, j}^{r}\right)\left(l_{u, j}^{r}\right)^{\frac{1-\alpha}{\alpha}}}{\sum_{u \in \boldsymbol{U}} \sum_{r \in \boldsymbol{R}}\left(x_{u, j}^{r}+d_{u, j}^{r}\right)\left(l_{u, j}^{r}\right)^{\frac{1-\alpha}{\alpha}}}, \forall j \in \boldsymbol{J} .
$$

Proof: Given the binary user association for the tuple (u,j,r), the optimization problem in 14 is convex with respect to the variable $\delta_{u, j}^{r}$. The equivalent Lagrangian function of the (14) can be defined as follows.

$$
\begin{gathered}
L=-\sum_{u \in \boldsymbol{U}} \frac{\left(\sum_{r \in \boldsymbol{R}} \sum_{j \in \boldsymbol{J}} x_{u, j}^{r} l_{u, j}^{r} \delta_{u, j}^{r}+\sum_{r \in \boldsymbol{R}} \sum_{k \in \boldsymbol{J}} d_{u, k}^{r} l_{u, k}^{r} \delta_{u, k}^{r}\right)^{1-\alpha}}{1-\alpha} \\
+\sum_{j \in \boldsymbol{j}} \mathbf{Y}_{j}\left(\sum_{u \in \boldsymbol{U}} \sum_{r \in \boldsymbol{R}}\left(x_{u, j}^{r}+d_{u, j}^{r}\right) \delta_{u, j}^{r}-1\right) \\
+\sum_{j \in \boldsymbol{j}} \mathbf{W}_{j}\left(\sum_{u \in \boldsymbol{U}} \sum_{r \in \boldsymbol{R}}\left(x_{u, j}^{r}+d_{u, j}^{r}\right)-1\right) \\
-\sum_{u \in \boldsymbol{U}} \sum_{r \in \boldsymbol{R}} \sum_{j \in \boldsymbol{J}} \mathbf{Z}_{u, j}^{r} \delta_{u, j}^{r}
\end{gathered}
$$

where the $\mathbf{Y}_{j}, \mathbf{W}_{j}$, and $\mathbf{Z}_{u, j}^{r}$ are intermediate Lagrangian variables. The necessary KKT conditions to compute the optimal solution for the given problem are as follows.

$$
\begin{aligned}
\frac{d L}{d \delta_{u, j}^{r}} & =0, \\
\sum_{j \in \boldsymbol{J}} \mathbf{Y}_{j}\left(\sum_{u \in \boldsymbol{U}} \sum_{r \in \boldsymbol{R}}\left(x_{u, j}^{r}+d_{u, j}^{r}\right) \delta_{u, j}^{r}-1\right) & =0, \\
\mathbf{Z}_{u, j}^{r} \delta_{u, j}^{r} & =0,
\end{aligned}
$$

where, 29] is the first order necessary condition for optimal $\delta_{u, j}^{r}$. 30 and (31) are the complementary-slackness conditions. Applying (29) in 28), we get

$$
\begin{gathered}
-\left(x_{u, j}^{r}+d_{u, j}^{r}\right)\left(l_{u, j}^{r}\right)^{1-\alpha}\left(\delta_{u, j}^{r}\right)^{-\alpha}+\mathbf{Y}_{j}-\mathbf{Z}_{\mathbf{u}, \mathbf{j}}^{\mathbf{r}}=0 \\
\left(x_{u, j}^{r}+d_{u, j}^{r}\right)\left(l_{u, j}^{r}\right)^{1-\alpha}=\mathbf{Y}_{j}-\mathbf{Z}_{u, j}^{r},
\end{gathered}
$$

The optimal $\delta_{u, j}^{r}$ should satisfy the positivity condition $\delta_{u, j}^{r}>$ 0 as given in (21) and should follow the complementaryslackness condition as in (31). Therefore, we consider $\mathbf{Z}_{u, j}^{r}=$ 0 . Using $\mathbf{Z}_{u, j}^{r}=0$ in (33) and on further simplification,

$$
\delta_{u, j}^{r}=\frac{\left(x_{u, j}^{r}+d_{u, j}^{r}\right)\left(l_{u, j}^{r}\right)^{\frac{1-\alpha}{\alpha}}}{\mathbf{Y}_{j}^{\frac{1}{\alpha}}},
$$

Substituting (34) in the constraint (19), we get,

$$
\mathbf{Y}_{j}^{\frac{1}{\alpha}}=\sum_{u \in \boldsymbol{U}} \sum_{r \in \boldsymbol{R}}\left(x_{u, j}^{r}+d_{u, j}^{r}\right)\left(l_{u, j}^{r}\right)^{\frac{1-\alpha}{\alpha}}
$$

Substituting (35) in the constraint (34), we get (27), This completes the proof of Proposition 1.

Similarly, the optimal user scheduling time fraction can be derived for $\alpha=1$ and $\alpha \rightarrow \infty$. The user scheduling time fraction of user $u$ from BS $j$ via RIS $r$ for proportionally fair (PF) scheduler with $\alpha=1$ is given as

$$
\delta_{u, j}^{r}=\frac{\left(x_{u, j}^{r}+d_{u, j}^{r}\right)}{\sum_{u \in \boldsymbol{U}} \sum_{r \in \boldsymbol{R}}\left(x_{u, j}^{r}+d_{u, j}^{r}\right)}, \forall j \in \boldsymbol{J} .
$$

Further, when $\alpha \rightarrow \infty$, the user scheduler with max-min fairness for RIS assisted DC system is written as follows.

$$
\delta_{u, j}^{r}=\frac{\left(x_{u, j}^{r}+d_{u, j}^{r}\right)\left(l_{u, j}^{r}\right)^{-1}}{\sum_{u \in \boldsymbol{U}} \sum_{r \in \boldsymbol{R}}\left(x_{u, j}^{r}+d_{u, j}^{r}\right)\left(l_{u, j}^{r}\right)^{-1}}, \forall j \in \boldsymbol{J} .
$$




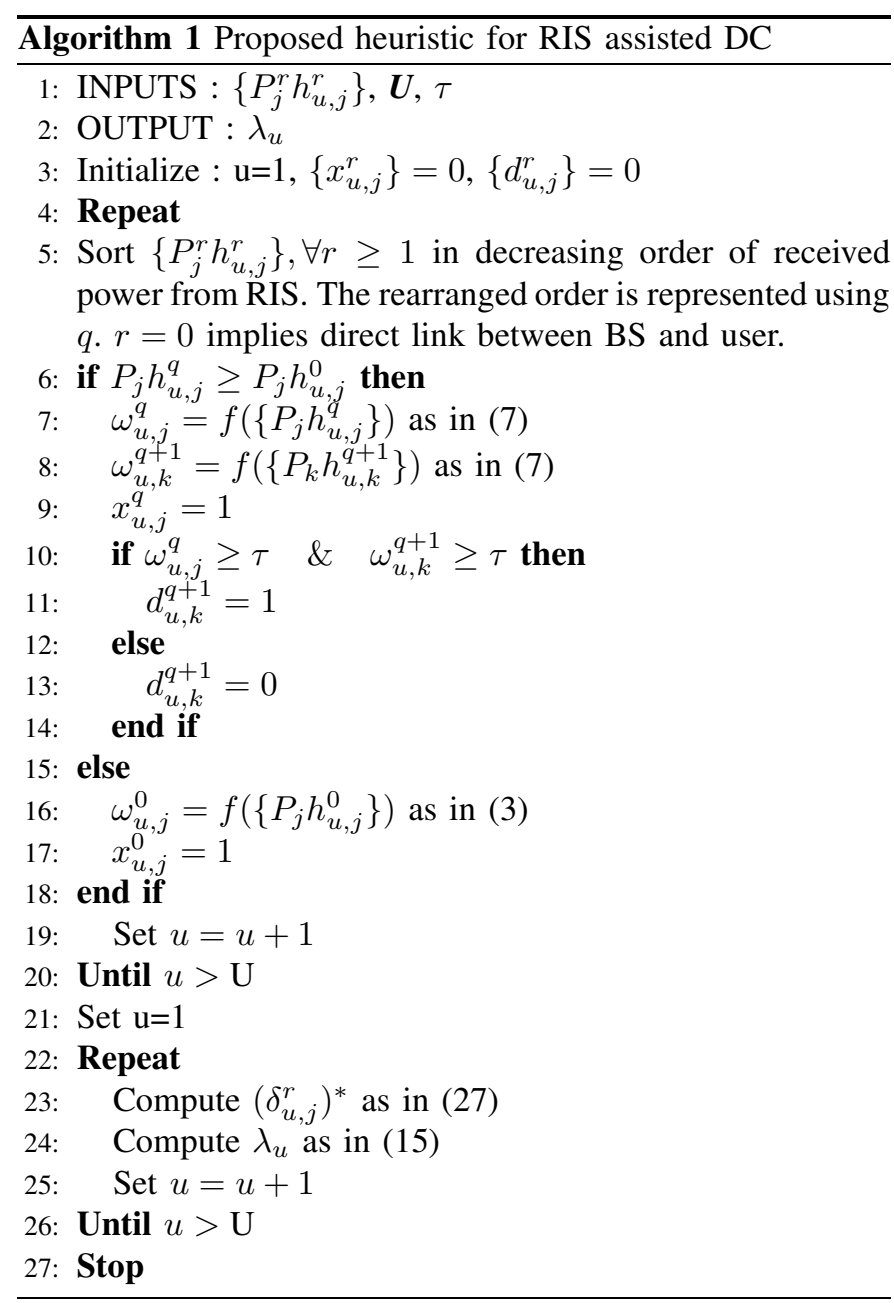

The heuristic for solving the given optimization problem with the derived optimal user scheduling time fraction is presented next.

\section{Proposed Heuristic For RIS Assisted DC}

In this section, we present the proposed heuristic for RIS assisted DC system as in (14). The problem presented in (14) is the global optimization problem for RIS assisted DC system. This involves the selection of DC users, scheduling, and computation of its resultant data rate. Solving for all at once is NP hard and is difficult to solve. We propose this heuristic for step by step procedure to solve the optimization problem proposed in (14). The set of received powers, user set, and the threshold for the selection of DC users $(\tau)$ are taken as inputs. The resultant data rate as in (15) is the output of the system. The heuristic is initialized by setting, $u=1$, binary association matrix $x_{u, j}^{r}=0$, and DC binary association matrix $d_{u, j}^{r}=0$. As an initial step, the received power of the user $u=1$ from all RIS is arranged in the decreasing order. The rearranged order of RIS based on the received power is now denoted with a variable $q$. The SINR of the user $u$ is computed using (7) or (3) based on its received power. If the received power of users' direct link with the BS is greater than the received power with RIS, then the SINR of the user $u$ is computed using (3) and the corresponding $x_{u, j}^{0}$ is set to 1 . If
TABLE III

SIMULATION PARAMETERS

\begin{tabular}{|c|c|}
\hline$f_{c}$ & $28 \mathrm{GHz}$ \\
\hline Penetration loss $\left(v_{s c}\right)$ & $20 \mathrm{~dB}$ for NLOS path \\
\hline Loss due to shadowing $(\rho)$ & Standard deviation of 4 dB \\
\hline $\mathrm{P}_{j}$ & $30 \mathrm{dBm}$ \\
\hline $\mathrm{PL}(\mathrm{d})$ & Urban micro $[23]$ \\
\hline$C$ & 99 \\
\hline Subchannel Bandwidth & $720 \mathrm{KHz}$ \\
\hline SC $_{\text {OFDM }}$ & 12 \\
\hline SY $_{\text {OFDM }}$ & 14 \\
\hline $\mathrm{T}_{\text {Subframe }}$ & $0.25 \mathrm{~ms}$ \\
\hline
\end{tabular}

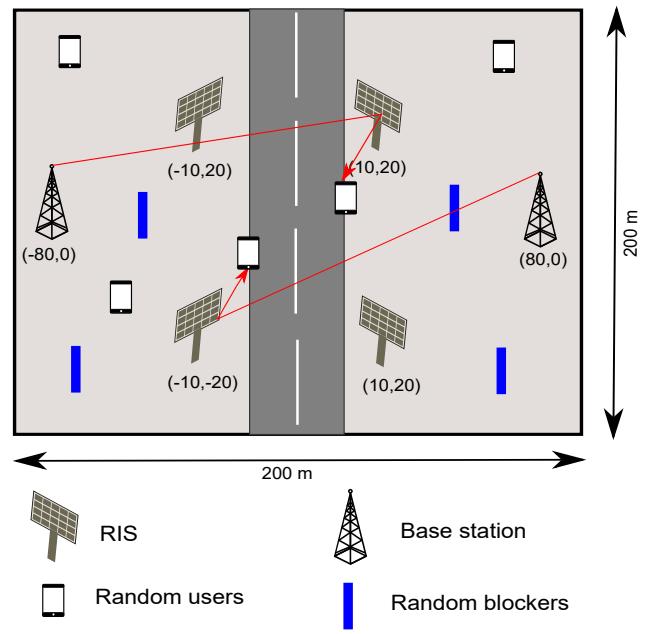

Fig. 2. Simulation settings

received power with RIS is greater than the direct link, then the SINR is computed using (7) and so the $x_{u, j}^{q}$ is set to 1 . Further, we define DC users as eligible users to utilize radio resource from two different BSs via two different RIS. If the first two values of users' received power in rearranged is greater than certain predefined threshold $\tau$, then the user is considered to be RIS assisted DC user. This user, now utilizes radio from two different BSs via two different RISs. Given all the SINR of users with direct and RIS link have been computed, the next step is to compute users' resultant data rate $\left(\lambda_{u}\right)$ as in (15). To compute $\lambda_{u}$, we need to first compute the optimal user scheduling time fraction $\left(\delta_{u, j}^{r}\right)^{*}$ as in (27). The complexity of the heuristic is in the order of $2 \mathrm{U}$, where $\mathrm{U}$ is the total number of users in the system. The heuristic is divided into two main steps. The computation of SINR and the selection of normal, DC, and RIS assisted DC users at the first step. The latter step continues with computation of optimal user scheduling time fraction and the resultant data rate. The numerical evaluation for different scenarios are presented next.

\section{Numerical Results}

We consider an area of $200 \mathrm{~m}$ x $200 \mathrm{~m}$. We consider deterministic BSs and RIS locations. This is acceptable because, the RIS is determined to be deployed based on the BS locations and its associated high blockage loss places. The users and blockers are deployed uniformly randomly in the considered 


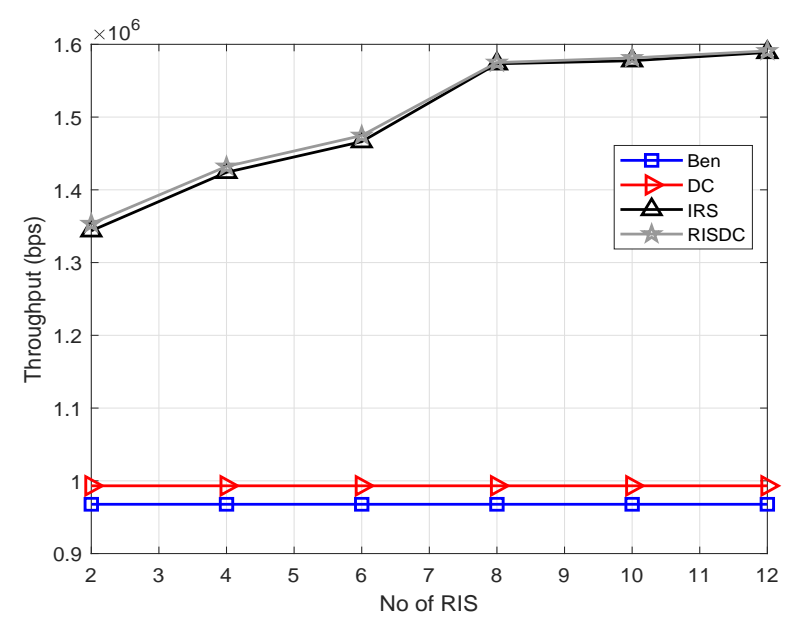

Fig. 3. Variation of throughput with respect to number of RISs when $\rho_{b s}=2$, $\rho_{\text {user }}=60$, and $\mu_{b}=10$.

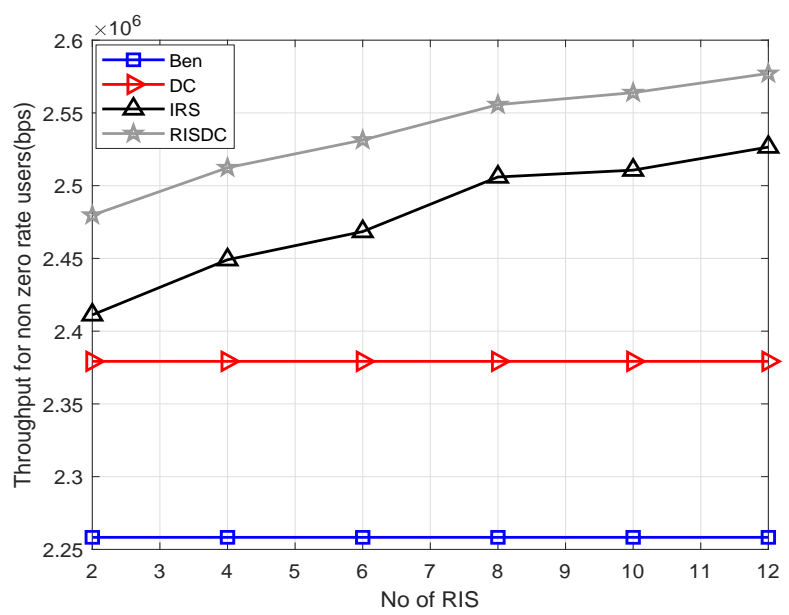

Fig. 4. Variation of throughput of non-zero rate users (users only in coverage) with respect to number of RISs when $\rho_{b s}=2, \rho_{\text {user }}=60$, and $\mu_{b}=10$.

area. The results of throughput are averaged over $10^{4}$ independent location realizations. The simulation parameters are listed in Table. IIII The channel between BS and user is considered to be NLOS and its associated pathloss is computed from [23]. We evaluate the performance of mmWave based cellular system. However, given the channel, the derived results are applicable for any system in general. We consider evaluating the coverage and throughput performance four systems namely, benchmark (Ben), DC, RIS, and RIS assisted DC (RISDC). The benchmark system is the conventional mmWave based cellular system without DC and RIS technologies. In DC, an eligible user can able to utilize radio resources from two different BSs directly. Since mmWave based cellular systems are prone to high blockage and attenuation, a controllable path with RIS has been considered. The eligible RIS users are served via RIS by the associated BSs. To further improve the per users' rate, the proposed RISDC system leverage the

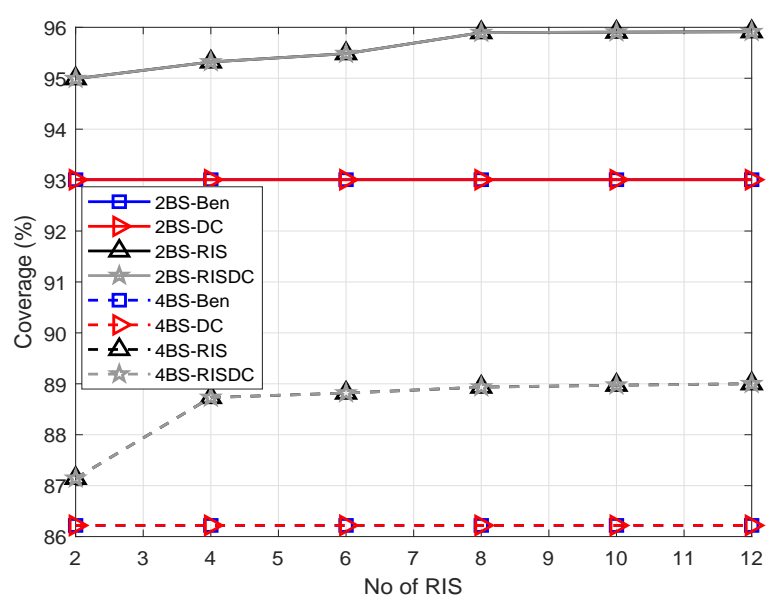

Fig. 5. Variation of coverage with respect to number of RISs when $\rho_{b s}=$ $[2,4], \rho_{u s e r}=60, \alpha=1$, and $\mu_{b}=10$

advantage of DC in a RIS based system. In this RISDC system, the eligible to be served as RISDC users are identified and then RIS based users are also selected based on the predetermined SINR threshold. RISDC system consists of both RISDC and RIS users users. Please note, based on the formulation and user selection, the RISDC users are the subset of RIS users, i.e., The user will be RISDC only if it's two associated RIS links' SINR are greater than certain predetermined threshold $\tau$. The coverage, RIS user selection and RISDC user selection threshold are set to be $-6.5 d B$ [21] throughout the simulation. However, these values can be varied based on the scenario and derived results remains the same for all the scenarios. The throughput corresponding to the (14) is given as follows.

$$
T_{\alpha}= \begin{cases}\left(\frac{1}{\mathrm{U}} \sum_{u \in \boldsymbol{U}} \lambda_{u}^{1-\alpha}\right)^{\frac{1}{1-\alpha}} & , \alpha>0, \alpha \neq 1, \\ \left(\prod_{u \in \boldsymbol{U}} \lambda_{u}\right)^{\frac{1}{\mathrm{U}}} & , \alpha=1,\end{cases}
$$

where, $\alpha$ is the fairness parameter, $\lambda_{u}$ is as defined in (15), and $\boldsymbol{U}$ is the set of users in the system. To study the impact of distributed RIS, the number of RIS associated to BS are varied. The impact on throughput and coverage by varying user and BS densities. Further, the user density is varied from 20 to 120 users in the considered area $\left(0.04 / \mathrm{km}^{2}\right)$ to see the performance of RIS assisted DC with different user densities. The simulation parameters are specified in Table. [III and a snapshot of simulation settings is shown in Fig. 2

We follow snapshot based approach, where the users and blockages are distributed uniformly randomly in the considered area. The simulation is carried out using MATLAB R2020b. We consider throughput for all the users in the system and throughput for non zero rate users in the system. The non zero rate users are defined as the users under coverage by the BSs (its $S I N R \geq-6.5 \mathrm{~dB}$ ). The variation of throughput with respect to number of RIS in the system is shown in Fig. 3 The proposed RISDC systems performs better than the benchmark 


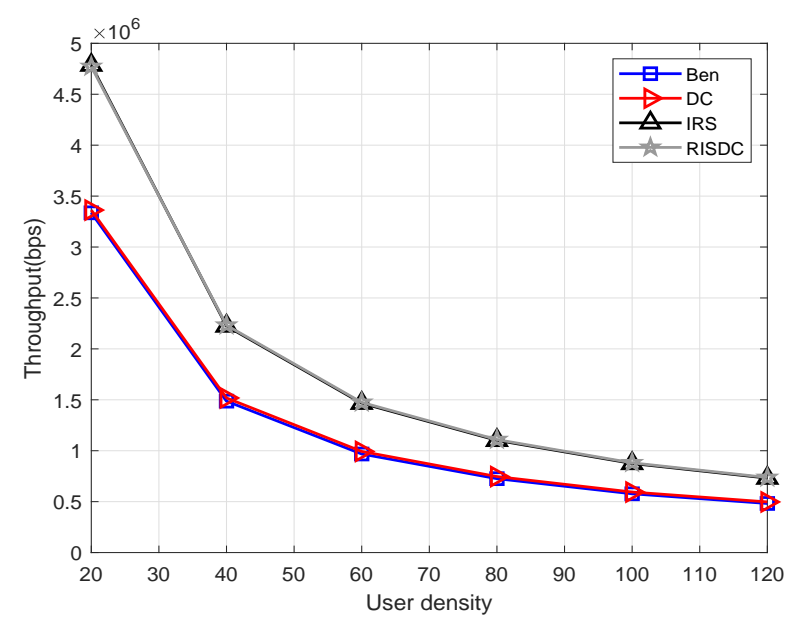

Fig. 6. Variation of throughput with respect to number of users when $\rho_{b s}=2$, $\rho_{\text {ris }}=6, \alpha=1$, and $\mu_{b}=10$.

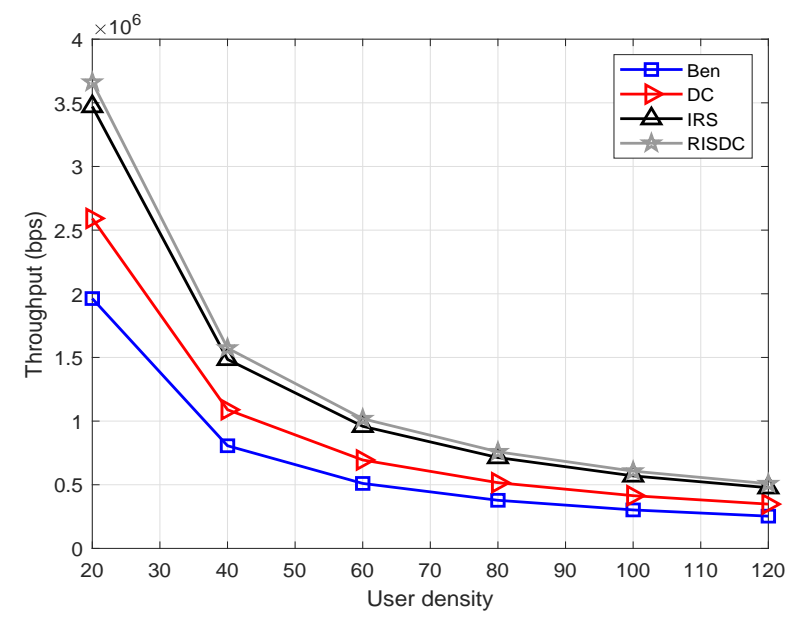

Fig. 7. Variation of throughput with respect to number of users when $\rho_{b s}=4$, $\rho_{\text {ris }}=6, \alpha=1$, and $\mu_{b}=10$.

and DC system in Fig. 3 with the gain of approximately 52\% and $48 \%$, respectively. But, the RISDC performs in similar to the RIS with a gain of $0.56 \%$ when we consider throughput of all users. However, if we consider only the users in coverage, the gain due to RISDC is comparably higher than the RIS system which is approximately $2.5 \%$. This is due to the fact that the throughput in (38) for $\alpha=1$ is geometric mean rate. The geometric mean rate is better when all the users' rate are better. Therefore, in order to observe the gain of RISDC system when compared to the other systems, the throughput for only users in coverage (non-zero rate users) are computed and shown in Fig. 4 The users with $S I N R \geq-6.5 d B$ are only in coverage and have non-zero rate according to Table. II The significant gain of the RISDC system when compared to other benchmark (12\%), DC(6\%), and RIS (2.4\%) for system considering only non zero rate users is observed in Fig. (4.

The coverage of the system with varying number of RISs

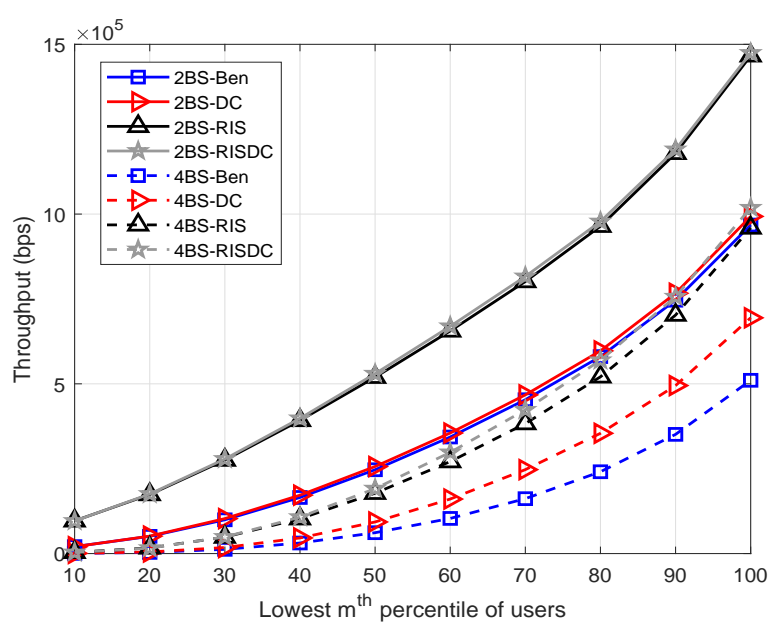

Fig. 8. Variation of throughput with respect to lowest $m^{\text {th }}$ percentile of users when $\rho_{b s}=[2,4]$, $\rho_{u s e r}=60, \alpha=1$, and $\mu_{b}=10$.

and the user density of $\rho_{\text {user }}=60$ for different number of BSs are shown in Fig. 5. We evaluated for 2 mmWave based BSs (2BS) and 4 mmWave based BSs (4BS) deployed in the same area. The coverage in Fig. 5 is computed over $10^{4}$ iterations. Each time the number of users in coverage are computed and averaged over $10^{4}$ realizations. The coverage with more number of BSs is less than the coverage with less number of BSs. This is because, as the number of BSs in the system increases, the interference also increases which substantially reduces the SINR of the users. In this, the coverage with RIS and RISDC is also less because we consider interference from other BSs for RIS and RISDC users. Further, the throughput performance with varying user densities for different number of BSs in the systems are shown in Fig. 6 and Fig. 7. Since all the users in the system are considered for computing throughput, the gain that we observe from Fig. 6 and Fig. 7 is minimal. If we consider throughput of the users in coverage, then, a significant gain is achieved with RISDC system. However, the throughput with 4BSs in Fig. 7 is less than the 2BS case as in Fig. 6 because it becomes interference limited system when the number of BSs increases.

The users' rate are arranged based on the increasing order. We consider evaluating the throughput for $\alpha=1$ in (38) for every $10 \%$ of users. This is defined as lowest $m^{\text {th }}$ percentile of users. This $m$ is varied as every $10 \%$ as shown in Fig. 8 . The same interference limited scenario for increasing number of BSs is observed while computing this worst case users' throughput also. There is a small gain achieved by RISDC system but the performance is similar when compared to RIS system. Those increase in comparable throughput is maintained even with varying the blockage density in the system as shown in Fig. 9. As the average number of blockage $\left(\mu_{b}\right)$ in the system increases, the gain dude direct LOS decreases significantly, which in turn reduces the throughput of the system. However, the gain of the proposed RISDC system with respect to the RIS system as $0.56 \%, 0.94 \%, 5.19 \%$, and $11.8 \%$ for blockage densities of $10,20,30$, and 40 , respectively. 


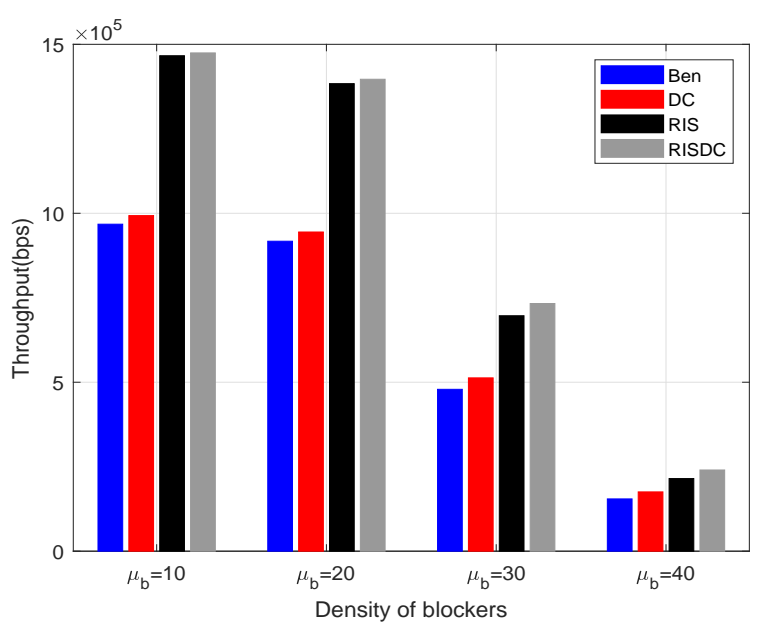

Fig. 9. Variation of throughput with respect to blockage density $\mu_{b}$ when $\rho_{b s}=2, \rho_{u s e r}=60, \rho_{\text {ris }}=6$, and $\alpha=1$.

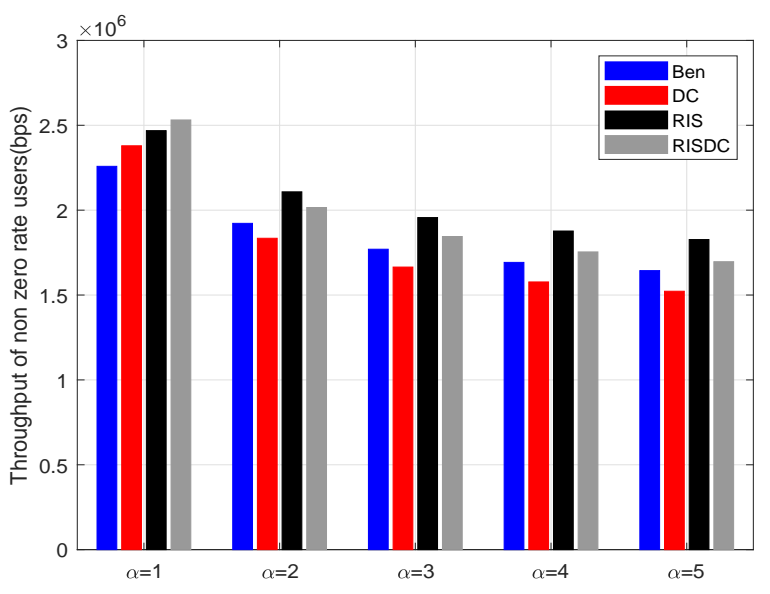

Fig. 10. Variation of non zero rate users' throughput for different $\alpha$ s when $\rho_{b s}=2, \rho_{\text {user }}=60, \rho_{\text {ris }}=6$, and $\mu_{b}=10$.

The proposed RISDC system is for a $\alpha$-fair utility function. While the $\alpha$ increases, the fairness to the worst case users' rate increase at the cost of best case user's rate. As a result, the throughput of users in coverage starts decreasing as in Fig. 10. Further, when $\alpha \geq 2$, the benchmark (Ben) and RIS throughput surpasses the DC and RISDC system's throughput, respectively. As fairness in the system $(\alpha)$ increases, the users' rate due to DC in benchmark and RIS system saturates as there is only limited resource available. Hence, RIS and RISDC system improves the coverage and data rate of the user instead of throughput of the system when $\alpha \geq 2$. This improvement in data rate of the proposed RISDC system leads to small gain in terms of throughput but the gain does not change considerably. However, the proposed $\alpha$-fair scheduling for RIS assisted DC outperforms other benchmark and DC system in terms of throughput for various BS, user, and blockage densities.

\section{CONCLUSiOn AND Future Work}

The RIS assisted DC system is proposed by utilizing the RIS resource and radio resource from two different RIS and BS, respectively. The joint resource allocation for RIS assisted DC system is formulated as an optimization problem and the user scheduling time fraction is derived for an $\alpha$-fair scheduler. Further, a heuristic for solving given RIS assisted DC problem with the derived optimal user scheduling time fractions is presented. The exhaustive simulation results of throughput are presented for different user, BS, and blocker densities. The proposed system provides significant throughput gain of $50 \%$ and $48 \%$ in certain scenarios when compared to the existing benchmark and DC systems. It is also shown that the coverage with increased number of BSs in the system decreases and saturates beyond certain RIS density. For RIS to be advantageous as standalone and RIS assisted DC system, the RIS deployment should be carefully designed for any BS. In future, we consider evaluating the performance of various RIS assisted wireless systems during uplink.

\section{REFERENCES}

[1] "5G Evolution and 6G,"Japan, NTT DOCOMO, INC, White Paper, 2021.

[2] W. Tang et al., "Wireless Communications With Reconfigurable Intelligent Surface: Path Loss Modeling and Experimental Measurement," in IEEE Transactions on Wireless Communications, vol. 20, no. 1, pp. 421439, Jan. 2021, doi: 10.1109/TWC.2020.3024887.

[3] M. Di Renzo et al., "Smart Radio Environments Empowered by Reconfigurable Intelligent Surfaces: How It Works, State of Research, and The Road Ahead," in IEEE Journal on Selected Areas in Communications, vol. 38, no. 11, pp. 2450-2525, Nov. 2020, doi: 10.1109/JSAC.2020.3007211.

[4] Ö. Özdogan, E. Björnson and E. G. Larsson, "Intelligent Reflecting Surfaces: Physics, Propagation, and Pathloss Modeling," in IEEE Wireless Communications Letters, vol. 9, no. 5, pp. 581-585, May 2020, doi: 10.1109/LWC.2019.2960779.

[5] M. A. ElMossallamy, H. Zhang, L. Song, K. G. Seddik, Z. Han and G. Y. Li, "Reconfigurable Intelligent Surfaces for Wireless Communications: Principles, Challenges, and Opportunities," in IEEE Transactions on Cognitive Communications and Networking, vol. 6, no. 3, pp. 990-1002, Sept. 2020, doi: 10.1109/TCCN.2020.2992604.

[6] H. Xie, J. Xu and Y. -F. Liu, "Max-Min Fairness in RIS-Aided MultiCell MISO Systems With Joint Transmit and Reflective Beamforming," in IEEE Transactions on Wireless Communications, vol. 20, no. 2, pp. 1379-1393, Feb. 2021, doi: 10.1109/TWC.2020.3033332.

[7] C. Pan et al., "Multicell MIMO Communications Relying on Intelligent Reflecting Surfaces," in IEEE Transactions on Wireless Communications, vol. 19, no. 8, pp. 5218-5233, Aug. 2020, doi: 10.1109/TWC.2020.2990766.

[8] Z. Li, M. Hua, Q. Wang and Q. Song, "Weighted Sum-Rate Maximization for Multi-RIS Aided Cooperative Transmission," in IEEE Wireless Communications Letters, vol. 9, no. 10, pp. 1620-1624, Oct. 2020, doi: 10.1109/LWC.2020.2999356

[9] X. Mu, Y. Liu, L. Guo, J. Lin and R. Schober, "Joint Deployment and Multiple Access Design for Intelligent Reflecting Surface Assisted Networks," in IEEE Transactions on Wireless Communications, doi: 10.1109/TWC.2021.3075885.

[10] J. Zuo, Y. Liu, Z. Qin and N. Al-Dhahir, "Resource Allocation in Intelligent Reflecting Surface Assisted NOMA Systems," in IEEE Transactions on Communications, vol. 68, no. 11, pp. 7170-7183, Nov. 2020, doi: 10.1109/TCOMM.2020.3016742

[11] M. Hua, Q. Wu, D. W. K. Ng, J. Zhao and L. Yang, "Intelligent Reflecting Surface-Aided Joint Processing Coordinated Multipoint Transmission," in IEEE Transactions on Communications, vol. 69, no. 3, pp. 1650-1665, March 2021, doi: 10.1109/TCOMM.2020.3042275.

[12] Y. Chen, Y. Wang, J. Zhang and Z. Li, "Resource Allocation for Intelligent Reflecting Surface Aided Vehicular Communications," in IEEE Transactions on Vehicular Technology, vol. 69, no. 10, pp. 12321-12326, Oct. 2020, doi: 10.1109/TVT.2020.3010252. 
[13] A. Zappone, M. Di Renzo, X. Xi and M. Debbah, "On the Optimal Number of Reflecting Elements for Reconfigurable Intelligent Surfaces," in IEEE Wireless Communications Letters, vol. 10, no. 3, pp. 464-468, March 2021, doi: 10.1109/LWC.2020.3034686.

[14] Y. Yang, S. Zhang and R. Zhang, "RIS-Enhanced OFDMA: Joint Resource Allocation and Passive Beamforming Optimization," in IEEE Wireless Communications Letters, vol. 9, no. 6, pp. 760-764, June 2020, doi: 10.1109/LWC.2020.2968303.

[15] Y. Yang, B. Zheng, S. Zhang and R. Zhang, "Intelligent Reflecting Surface Meets OFDM: Protocol Design and Rate Maximization," in IEEE Transactions on Communications, vol. 68, no. 7, pp. 4522-4535, July 2020, doi: 10.1109/TCOMM.2020.2981458.

[16] Y. Guo, Z. Qin, Y. Liu and N. Al-Dhahir, "Intelligent Reflecting Surface Aided Multiple Access Over Fading Channels," in IEEE Transactions on Communications, vol. 69, no. 3, pp. 2015-2027, March 2021, doi: 10.1109/TCOMM.2020.3042277.

[17] H. Du, J. Zhang, J. Cheng and B. Ai, "Millimeter Wave Communications With Reconfigurable Intelligent Surfaces: Performance Analysis and Optimization," in IEEE Transactions on Communications, vol. 69, no. 4, pp. 2752-2768, April 2021, doi: 10.1109/TCOMM.2021.3051682.

[18] 3GPP, "Overview of 3GPP Release 12," 3GPP RP 151570, V0.2.0, September, 2015.

[19] M. S. Pan, T. M. Lin, C. Y. Chiu, and C. Y. Wang, "Downlink traffic scheduling for LTE-A small cell networks with dual connectivity enhancement," IEEE Communications Letters, vol. 20, no. 4, pp. 796799, April 2016.

[20] Y. Ramamoorthi and A. Kumar, "Performance comparison of dual connectivity with CoMP in heterogeneous cellular networks,"2017 9th International Conference on Communication Systems and Networks (COMSNETS), 2017, pp. 237-242, doi: 10.1109/COMSNETS.2017.7945382.

[21] Y. Ramamoorthi and A. Kumar, "Resource Allocation for CoMP in Cellular Networks With Base Station Sleeping," IEEE Access, vol. 6, pp. 12620-12633, March 2018.

[22] E. Björnson, Ö. Özdogan and E. G. Larsson, "Intelligent Reflecting Surface Versus Decode-and-Forward: How Large Surfaces are Needed to Beat Relaying?," in IEEE Wireless Communications Letters, vol. 9, no. 2, pp. 244-248, Feb. 2020, doi: 10.1109/LWC.2019.2950624.

[23] 3GPP, "Technical Specification Group Radio Access Network; Study on channel model for frequencies from 0.5 to $100 \mathrm{GHz}$," 3rd Gener. Partnership Project (3GPP), Tech. Rep. TR 38.901, v16.1.0, 2019.

Yoghitha Ramamoorthi received the B.E. and M.E. from Anna University, Chennai, India in 2012 and 2014, respectively. She received Ph.D. from the Department of Electrical Engineering, Indian Institute of Technology, Hyderabad in 2020. She is currently a Postdoctoral researcher in NTT Access Network Service Systems Laboratories, Yokosuka, Japan. Her research interests include intelligent reflecting surfaces, energy efficiency, resource allocation, and multi-connectivity technologies of next-generation wireless networks.

Masashi Iwabuchi received the B.S. and M.S. degrees from the Tokyo Institute of Technology, Tokyo, Japan, in 2008 and 2010, respectively. From 2010 to 2016, he joined the NTT Access Network Service Systems Laboratories, Nippon Telegraph and Telephone Corporation (NTT). From 2016 to 2019, he joined NTT DOCOMO, Inc. Since 2019, he has been with NTT again. His current interests are in smart repeaters and reconfigurable intelligent surfaces technologies for $5 \mathrm{G}$ evolution and $6 \mathrm{G}$. He received the Young Engineers Award from the Institute of Electronics, Information and Communication Engineers (IEICE) in 2015. He is a member of the IEICE.
Tomoki Murakami (Member, IEEE) received the B.E., M.E., and Dr.Eng. degrees from Waseda University, Japan in 2006, 2008, and 2015, respectively. In 2008, he joined NTT Network innovation Laboratories, Nippon Telegraph and Telephone Corporation (NTT), Yokosuka, Japan. He is currently a Research Engineer in the Wireless Access Systems Project. His current research interests are high efficiency technologies for future wireless systems. He received the Young Engineer Award from the IEICE in 2010, the Active Research Award from IEICE AP (Antenna and Propagation) in 2010, the Best Tutorial Paper Award from IEICE Communicaions society in 2014, the Best Paper Award and the KIYASU-Zen'iti Award from the IEICE in 2015, and the Best Paper Award from IEICE on AP in 2016. He is a member of IEEE and IEICE.

Tomoaki Ogawa received a B.A. in environmental information and an M.A. in media and governance from Keio University, Kanagawa, in 1996 and 1998. He joined NTT Network Innovation Laboratories in 1998, where he has been working on indoor-location systems. His recent interest focuses on development of wireless local area network services. He is a member of IEICE.

Yasushi Takatori (Member, IEEE) received the B.E. degree in electrical and communication engineering and the M.E. degree in system information engineering from Tohoku University, Miyagi, in 1993 and 1995, respectively. $\mathrm{He}$ received the $\mathrm{Ph} . \mathrm{D}$. degree in wireless communication engineering from Aalborg University, Aalborg, Denmark, in 2005. He joined NTT in 1995. He is currently working on R\&D of high efficiency wireless access systems as well as the optical core network. He served as a co-chair of COEX Ad Hoc in IEEE 802.11ac from 2009 to 2010. He was a visiting researcher at the Center for TeleInFrastruktur, Aalborg University, Denmark, from 2004 and 2005. He received the Young Engineer Award from IEICE in 2000 and the Best Paper Award of IEICE in 2011. He is a senior member of IEICE and a member of IEEE. 